\title{
Pinning down a moving target: A standard HIV
}

ANNAPOLIS, MD and SAN FRANCISCO, CA-The problem with the human immunodeficiency virus (HIV) - or one of them, at least-is that it represents a moving target for researchers. Because of its high rate of replication and an error-prone, reverse transcriptase-based, mechanism for reproducing, HIV is particularly adept at producing variants. And the problem is only going to get worse: The use of new antivirals drugs-and combinations of those drugs-will put selective pressure on HIV for change. As John Coffin of Tufts University Medical School (Boston, MA), who spoke during the opening symposium of the 35th Interscience Conference on Antimicrobial Agents and Chemotherapy ICAAC), held in September in San Francisco pointed out, "For no antiviral strategy so far has HIV been unable to generate resistant mutants."

At one time, zidovudine (AZT) was the only drug approved specifically for combating AIDS. Now clinical researchers are reporting that combinations of various nucleotide-based reverse transcriptase (RT) inhibitorssometimes also administered with even newer inhibitors of another HIV target, the viral protease-are superior to AZT. While this is good news, the pressing need is to confront HIV's uncanny ability to produce drug-resistant variants.

HIV's high mutation rate and its resistance to therapeutic agents is not just an academic issue. Officials at the U.S. Food and Drug Administration (FDA, Rockville, MD) are insisting that companies furnish the agency with information on HIV resistance for new antiviral products undergoing review. Such information may be incorporated into the insert and label describing a product's appropriate clinical uses-a prospect that heightens anxieties for companies developing these products. At the Fourth Workshop on Viral Resistance, held in Annapolis, MD during September, participants including industry representatives agreed that new, more reliable as- says and reagents for assessing HIV resistance to antiviral agents are needed.

When HIV's drug resistance first arose, FDA officials developed guidelines to address what was then a relatively uncomplicated problem. But now, according to Michael Ussery of the FDA Division of Antiviral Products, "the guidelines ["Points to Consider in the Preclinical Development of Antiviral Drugs"] need to be revised." "We're trying to tailor our advice as best we can," he says. Some across-the-board changes may be incorporated into the agency's recommendations for monitoring resistance during the preclinical phase of antiviral drug development. But Ussery says that the main emphasis will be on talking to individual companies about specific products.

Ussery and other FDA officials are less certain about the information that should be developed to guide physicians who are treating HIV-infected patients. Companies researchers developing new agents are reluctant to see FDA move too quickly on this issue. They note that HIV's susceptibility to antiviral products is part of a dynamic, very complex, process. This complexity is likely to grow considerably as antivirals are administered to individual patients in increasingly baroque combinations. Some strategies involve the administration of several RT inhibitors simultaneously or the combination of one or several RT inhibitors with protease inhibitors. Yet other strategies call for intermittent use of immune system-stimulating products, notably interleukin-2 (IL-2).

To address such fundamental issues, investigators throughout this field need standard versions of HIV-both laboratory strains and clinical isolates-to use when assessing drug resistance, researchers at the Annapolis workshop concluded. Although countless strains of HIV that might be used, they all tend to change significantly each time they replicate. With that instablity superimposed on an already-bewilder- ing genetic variety, researchers find it difficult to pin down the common characteristics responsible for HIV's resistance to specific drugs being tested.

One approach to producing standardized versions of HIV, according to John Mellors of the University of Pittsburgh Medical Center (Pittsburgh, PA), would be to wrest the control of replication from the virus. Incorporating the HIV genome into a plasmid and multiplying the plasmid in a suitable host, for instance, would put HIV replication under the control of a more reliable enzymatic apparatus allowing, the HIV genome to be produced with greater fidelity.

The opening of new therapeutic avenues for treating HIV infections is also eroding the usefulness of the current "consensus protocol" used for measuring HIV resistance to antiviral agents. When only a single RT inhibitor was being used, researchers who followed the protocol obtained reliable results. "AZT was easy," says Douglas Mayers of the Walter Reed Army Institute of Research (Rockville, MD). But additional RT inhibitors brought new resistance patterns and, he says, "life became much more difficuit." Following the protocol gave results that were far more erratic than before. The consensus protocol "may just not work" for all RT inhibiting dugs, according to Mayers. "We either need to find a new assay or to get new intelligence about the mutations responsible for resistance to other antiviral agents," he says.

If the consensus protocol's continued usefulness seems in doubt, the situation is far worse for measuring resistance among other classes of antiviral agents, particularly HIV protease inhibitors. "The protease inhibitors are very, very complex," says Dean Winslow of DuPont Merck Pharmaceutical Company (Wilmington, DE). For example, mutations that occur outside the coding region for this viral gene product apparently can affect drug-resistance patterns. "We don't understand how these background changes affect the susceptibility and viability of mutant viruses," says Winslow.

— Jeffrey L. Fox
The

complexity [of HIV

resistance] is

likely to

grow

considerably

as antivirals

are given in

increasingly

baroque

combinations. 